\title{
Effects of Silk Maturity and Pollination on Infection of Maize Ears by Ustilago maydis
}

\author{
Lindsey J. du Toit and Jerald K. Pataky, Department of Crop Sciences, University of Illinois, Urbana 61801
}

\begin{abstract}
du Toit, L. J., and Pataky, J. K. 1999. Effects of silk maturity and pollination on infection of maize ears by Ustilago maydis. Plant Dis. 83:621-626.

Host resistance is the most efficient method of controlling common smut of maize (Zea mays), caused by Ustilago maydis. Precise timing of ear inoculations with $U$. maydis relative to silk maturity and pollination may improve the ability to screen maize germ plasm for resistance. The objectives of this study were to determine the length of time maize kernels can be infected by $U$. maydis through silks, and to examine the effects of pollination on infection through silks. Two field studies were done in 1995, 1996, and 1997 at the University of Illinois South Farms. In the date-of-inoculation study, ears were inoculated at 2- to 3-day intervals from early silk emergence until 16 days after silk emergence. In the date-of-planting study, hybrids were planted on four dates and ears were inoculated on the same day for all planting dates. In each study, ear shoots were covered with shoot bags prior to silk emergence to prevent pollination, or ear shoots were left uncovered to allow silks to be pollinated normally. Maize ears were susceptible to infection by $U$. maydis from silk emergence until 8 to 14 days after silk emergence. During this period of susceptibility, incidence of ears with galls decreased as silks aged. Incidence of ears with galls on plants inoculated 7 days apart differed by as much as $70 \%$. The period that maize ears were susceptible to infection by $U$. maydis was shorter and incidence of ears with galls decreased more rapidly when silks were exposed to pollen than when silks were not exposed. The silk channel method of inoculating for common smut does not appear to be practical for large-scale evaluations of numerous lines. The method is practical for evaluating a limited number of lines or for inducing ear galls for commercial production of huitlacoche (smut galls eaten at an immature stage).
\end{abstract}

Common smut of maize (Zea mays L.), caused by Ustilago maydis (DC.) Corda (= $U$. zeae (Beckm.) Unger), occurs sporadically in areas of maize production throughout the world (14). Losses due to common smut are associated mainly with ear galls (1). Smut causes greater economic damage to sweet corn than to other types of maize because an ear of sweet corn with only one or two galls is non-marketable, and because smutted ears create additional costs during harvest and processing. Host resistance is the most efficient method of controlling common smut, but resistance and the nature of the host-pathogen interaction are poorly understood, partly due to the lack of an efficient and reliable method of inoculating with $U$. maydis.

Maize ears are infected by $U$. maydis through silks, which extend from the ovaries and emerge through a silk channel formed by husk leaves at the tip of the ear.

Corresponding author: J. K. Pataky

E-mail: j-pataky@uiuc.edu

Present address of L. J. du Toit: Washington State University, 7612 Pioneer Way E, Puyallup 983714998.

Accepted for publication 19 March 1999.

Publication no. D-1999-0503-03R

(C) 1999 The American Phytopathological Society
Sporidia disseminated by wind or rain are deposited onto newly emerged silks (14). Compatible sporidia mate on the silk surface, forming a dikaryon which penetrates the silk by means of an appressorium. The fungus grows down the silk and into the developing ovary where galls form (15).

Maize silks undergo physiological change as they mature. Proteinaceous and pectic secretions accumulate on the surface of developing silks until senescence. Cells within the silk in the vicinity of the pollen tube pathway are also secretory, providing nutrition and guidance for pollen tube growth (7). Following successful pollination, an abscission zone forms at the base of the silk and the silk is shed (6), potentially precluding access to the fertilized ovary by pathogens that infect kernels via the silk.

The susceptibility of maize to pathogens that infect ears through silks varies with silk development. Ear rot caused by Fusarium graminearum Schwabe was greatest when silks were inoculated immediately after emergence, and declined rapidly with senescence of silks $(4,13)$. Kernel infection by Aspergillus flavus Link:Fr. was more severe when silks were inoculated at early senescence than at later stages of senescence (8). Kernels were colonized by $F$. moniliforme J. Sheld. soon after the onset of silk senescence (5).

Although silks have been inoculated with $U$. maydis at various stages from early silk emergence $(10,11,15,19,20)$ to the start of silk browning and senescence (12), the effect of aging on the receptivity of silks to infection by $U$. maydis has not been examined. Defining the period of time during which kernels can be infected by $U$. maydis through silks may improve efforts to screen for resistance, particularly in commercial breeding programs which evaluate hundreds of lines of varying maturity.

Snetselaar and Mims (15) hypothesized that ears remain susceptible to infection by $U$. maydis for a longer time when silks are not pollinated than when silks are pollinated, because $U$. maydis, an obligate biotroph in its dikaryotic form, is not able to grow across the layer of dead cells in the abscission zone of pollinated silks. Walter's (20) failure to observe ear infection by $U$. maydis may have been associated with his practice of supplying pollen to the silks during inoculation. Precise timing of ear inoculations with $U$. maydis relative to silk maturity and pollination may improve the ability to screen maize germ plasm for resistance to common smut.

The objectives of this study were to determine the period of time during which maize kernels can be infected by $U$. maydis through silks, and to examine the effects of pollination on infection through silks. Preliminary results have been reported (3).

\section{MATERIALS AND METHODS}

The susceptibility of maize ears to infection by $U$. maydis through silks of different ages and the effects of pollination on ear infection were examined in two field studies referred to as the date-of-inoculation study and the date-of-planting study. In the date-of-inoculation study, ears were inoculated at 2- to 3-day intervals from early silk emergence until 16 days after silk emergence. Thus, silk age was confounded with inoculation dates. To avoid confounding these two factors in the dateof-planting study, hybrids were planted on four dates and ears were inoculated on the same day for all planting dates.

There were two silk treatments in each study. Primary ear shoots were covered with shoot bags prior to silk emergence to prevent pollination or ear shoots were left uncovered to allow silks to be pollinated normally. Shoot bags were removed at the time of inoculation, allowing for pollination after inoculation.

Date-of-inoculation study. The experimental design was a split-plot, randomized 
complete block (RCB) with a 2-by-4 factorial treatment design in 1995 and a 2-by-8 factorial treatment design in 1996 and 1997. The treatment design in 1995 included the two silk treatments (ear shoots covered and ear shoots uncovered) and four silk ages determined by four dates of inoculation and measured as days after silk emergence. A total of 12 commercial sweet corn hybrids (Cabaret, Even Sweeter, Flagship, Florida Staysweet, GSS 4606, Pegasus, Sch 51092, Sch 81615, SS 7710, SS 8102, XPH 3024, and XPH 3056) were planted on 5 June 1995 on the University of Illinois South Farm. Hybrids were replicates in 1995. In 1996 and 1997, there were four replicates of 16 treatments for each of two sweet corn hybrids (Punchline and Dazzle). Hybrids were planted on 23 May 1996 and 13 May 1997 on the University of Illinois South Farm. Each hybrid was a separate experiment in 1996 and 1997. The treatment design included the two silk treatments and eight silk ages determined by eight inoculation dates and measured as days after silk emergence. Ear shoots in main plots were covered with shoot bags or left uncovered. Primary ears in split-plots were inoculated on one of eight days. An experimental unit was a 3row plot in 1995 and a 10-row plot in 1996 and 1997. Rows were $3 \mathrm{~m}$ long and spaced $0.76 \mathrm{~m}$ apart, with approximately 15 plants/row.

Inoculum was produced in potato dextrose broth (PDB) using the protocol of Snetselaar and Mims (15), with slight modifications. Inoculum was not centrifuged and resuspended in 1997 because these time-consuming procedures did not affect viability of inoculum (2). Sporidial suspensions of the two compatible isolates were diluted to approximately $10^{6}$ sporidia/ml and stored on ice for up to $3 \mathrm{~h}$ while plants were inoculated. Compatible isolates were mixed immediately prior to inoculation.

In 1995 , ears were inoculated $0,4,8$, or 12 days after silks had emerged about 1 to $2 \mathrm{~cm}$. Plants with silks not yet emerged or with silks emerged $3 \mathrm{~cm}$ or more by the first inoculation date, 6 August, were rogued. In 1996, the hybrid Punchline was inoculated 0,2 or $3,5,7,9,11,13$, and 14 or 15 days after silk emergence. Then hybrid Dazzle was inoculated 0, 3, 5, 7, 9, 11, 13 , and 16 days after silk emergence. In 1997, Punchline was inoculated 0, 2, 5, 7, $9,12,14$, and 16 days after silk emergence, and Dazzle was inoculated 0, 2, 4, 7, 9, 11, 14 , and 16 days after silk emergence. To ensure uniform maturity among inoculated plants, 50 plants with silks emerged 1 to 2 $\mathrm{cm}$ were tagged in each experimental unit the day prior to the first inoculation date. Tagged plants were inoculated on the appropriate day by injecting $3 \mathrm{ml}$ of a mixture of compatible sporidia of $U$. maydis down the silk channel of the primary ears. Ears were inoculated with a hog vaccinator (50-cc pistol grip vaco syringe, Model ID65, Ideal Instruments, Chicago) equipped with a needle modified by blocking the tip and drilling two small holes in the side for release of inoculum.

Inoculated ears with galls were counted in each experimental unit 3 to 4 weeks after inoculation. Incidence of ears with galls was calculated as a percentage: (number of ears with galls)/(total number of ears inoculated) $\times 100$. The analysis of variance (ANOVA) was calculated using Proc GLM of SAS (version 6.12; SAS Institute, Cary, NC). Data were transformed using the arcsin square root transformation to obtain independent, normally distributed residuals for the ANOVA (16). Regression equations describing the relationship between incidence and silk age were calculated from the first inoculation date until incidence was below $10 \%$, the level of natural infection in control plots. Trend lines were fitted to the relationship between incidence and silk age using Microsoft Excel (Microsoft, Redmond, WA).

Date-of-planting study. Field trials were planted in 1996 and 1997 as splitsplit-plot arrangements of a RCB design with four replications. The treatment design was a 4-by-4-by-2 factorial of four silk ages determined by four planting dates and measured as days after silk emergence, four sweet corn hybrids (Dazzle, Frontier, GH 2690, and Punchline) and two silk treatments (ear shoots covered with shoot bags and ear shoots left uncovered). Silk ages were the main plot treatments, hybrids were the split-plot treatments, and silk treatments were the split-split-plot treatments. An experimental unit was a 10-row plot in 1996 and an 8-row plot in 1997. Rows were $3 \mathrm{~m}$ long and spaced $0.76 \mathrm{~m}$ apart, with approximately 15 plants/row. Seed was planted on 24 May, 31 May, 5 June, and 13 June 1996; and on 13 May, 23 May, 4 June, and 17 June 1997.

In 1996, ears of all hybrids were inoculated on 7 August, approximately 3 days before silks had emerged 1 to $2 \mathrm{~cm}$ in the fourth planting date of the latest-maturing hybrid, GH 2690. Silk age ranged from 0 to 14 days after silk emergence for hybrid Dazzle, from 2 to 16 days after silk emergence for Frontier, from 3 days prior to silk emergence to 11 days after silk emergence for $\mathrm{GH} 2690$, and from 6 to 20 days after silk emergence for Punchline.

Hybrids were inoculated on different days in 1997 to time the first inoculation with early silk emergence of each hybrid. When silks of primary ears in each planting date had emerged 1 to $2 \mathrm{~cm}$ in any experimental unit, 40 to 50 plants of uniform maturity in that plot were tagged. Primary ears of tagged plants in all planting dates were inoculated on the day when silks had emerged 1 to $2 \mathrm{~cm}$ in the fourth planting date. Ears were inoculated on 4 August for the hybrid Punchline, 8 August for Dazzle and Frontier, and 13 August for
GH 2690. Silk age ranged from 2 days prior to silk emergence to 14 days after silk emergence for Dazzle, from 0 to 16 days after silk emergence for Frontier, from 1 to 18 days after silk emergence for GH 2690 , and from 1 to 15 days after silk emergence for Punchline.

Inoculated ears with galls were counted approximately 3 weeks after inoculation for each hybrid. Incidence of ears with galls was calculated as a percentage: (number of ears with galls)/(total number of ears inoculated) $\times 100$. The ANOVA and regression models describing the relationship between incidence and silk age were calculated using Proc GLM of SAS (version 6.12; SAS Institute). Trend lines were fitted to the relationship between incidence and silk age using Microsoft Excel.

\section{RESULTS}

Date-of-inoculation study. In 1995, incidence of ears with galls in inoculated plots ranged from 0 to $100 \%$ with a mean of $51 \%$ for ear shoots covered and $45 \%$ for ear shoots uncovered, although the main effect of silk treatments was not significant. The mean observed level of natural infection on uncovered ears of all hybrids was about $5 \%$ incidence of ears with galls. Incidence differed significantly with silk age and was $85,66,26$, and $7 \%$ when ears were inoculated $0,4,8$, and 12 days after silk emergence, respectively.

The interaction between silk age and silk treatments was significant. Incidence of ears with galls did not differ among silk treatments when ears were inoculated as silks emerged (85\% incidence) or when inoculated 12 days after silk emergence (7\% incidence; Fig. 1A). For plots in which ear shoots were covered, incidence decreased from 80 to $36 \%$ when plants were inoculated 4 and 8 days after silk emergence, respectively (Fig. 1A). For plots in which ear shoots were uncovered, incidence decreased from 66 to $26 \%$ when plants were inoculated 4 and 8 days after silk emergence, respectively (Fig. 1A). Thus, the reduction in incidence with silk age was delayed by about 2 days when ear shoots were covered. A linear regression model best described the relationship between incidence and silk age for uncovered ear shoots, accounting for $86 \%$ of the variation in incidence (Table 1). A quadratic regression model best described the decrease in incidence with silk age when ear shoots were covered, accounting for $86 \%$ of the variation in incidence (Table $1)$.

Results for 1996 and 1997 were pooled because interactions of years with hybrids, silk treatment, and silk age were not significant in the ANOVA combined over years. Results were analyzed separately for hybrids Dazzle and Punchline because of significant interactions of hybrids (experiments) with silk treatment and silk 
age in the ANOVA combined over hybrids. Incidence of ears with galls ranged from 0 to $100 \%$ with a mean of $45 \%$ for the hybrid Dazzle and $28 \%$ for Punchline. Incidence differed between silk treatments and among silk ages for both hybrids. The interaction between silk age and silk treat-

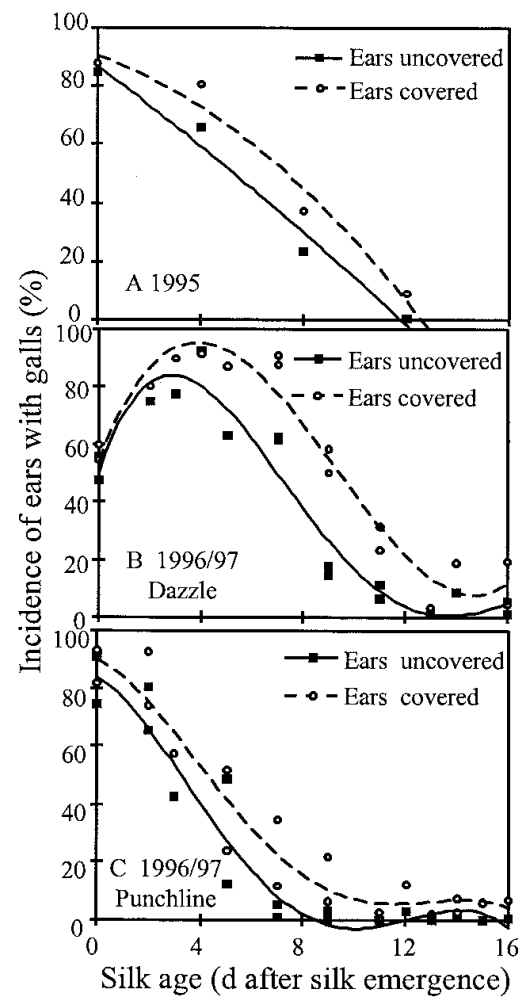

Fig. 1. Relationship between silk age and incidence of ears with galls of common smut on sweet corn when ear shoots were covered or uncovered prior to inoculation with Ustilago maydis (A) in 1995, and in 1996 and 1997 for hybrids (B) Dazzle and (C) Punchline, in the date-of-inoculation studies. Regression models are described in the text. Relationships are estimated by trend lines. Data points are the means of two years $(B, C)$. ment was significant for the hybrid Dazzle but not for Punchline.

For the hybrid Dazzle, ears inoculated 4 days after silk emergence had a higher incidence of galls than ears inoculated at silk emergence (Fig. 1B). Incidence decreased with silk age from 4 days after silk emergence until about 13 days after silk emergence, when incidence was $<10 \%$, the level associated with natural infection. Incidence did not differ between ears covered and ears uncovered when plants were inoculated 0 to 4 days after silk emergence, but was 15 to $30 \%$ greater for covered ears than for uncovered ears when plants were inoculated between 4 and 12 days after silk emergence (Fig. 1B). Covering ear shoots until inoculation delayed reduction in incidence by an amount equivalent to that which occurred in 2 to 3 days in uncovered plots of Dazzle (Fig. 1B). The cubic regression model described $77 \%$ of the variation in incidence with silk age from 0 to 13 days after silk emergence when ear shoots were uncovered (Table 1). The quadratic regression model described $77 \%$ of the variation in incidence from 0 to 13 days after silk emergence when ear shoots were covered (Table 1).

For the hybrid Punchline, incidence of ears with galls was greatest (about 85\%) when ears were inoculated as silks emerged. Incidence decreased with silk age to the level associated with natural infection (about 5\%) when plants were inoculated 8 to 11 days after silk emergence (Fig. 1C). Incidence was about $10 \%$ greater when ear shoots were covered than when ear shoots were uncovered. The reduction in incidence with silk age was delayed about 2 days when ear shoots of Punchline were covered (Fig. 1C). A linear model described $78 \%$ of the variation in incidence from 0 to 11 days after silk emergence (Table 1).

Date-of-planting study. Results for 1996 and 1997 were analyzed separately because of significant interactions of years with hybrids, silk age, and silk treatment in the ANOVA combined over years. In 1996, incidence of ears with galls ranged from 0 to $91 \%$. Incidence differed with silk age, among hybrids, and between silk treatments. Mean incidence of ears with galls was $50 \%$ for the hybrid Dazzle, $36 \%$ for Frontier, 26\% for GH 2690, and 6\% for Punchline. Mean incidence was $38 \%$ when ear shoots were covered and $22 \%$ when ear shoots were uncovered. Mean incidence was $40,42,24$, and $12 \%$ for the four silk ages, (youngest to oldest), as determined by the fourth, third, second, and first planting dates, respectively. Interactions among silk age, hybrids, and silk treatments were significant.

Incidence of ears with galls decreased with silk age for all hybrids in 1996 (Fig. $2 \mathrm{~A}$ to $\mathrm{D})$. The decrease in incidence was more rapid when ear shoots were uncovered prior to inoculation than when ear shoots were covered. The difference in incidence between covered and uncovered ear shoots ranged from 0 to $>50 \%$, with the greatest differences observed when ears were inoculated between 3 and 9 days after silk emergence (Fig. 2A to C).

For the hybrid Dazzle, incidence was $59 \%$ when covered ear shoots were inoculated at silk emergence and $85 \%$ when plants were inoculated 4 days after silk emergence (Fig. 2A). Incidence then decreased with silk age to $37 \%$, when covered ears were inoculated 14 days after silk emergence. A quadratic regression model described $50 \%$ of the variation in incidence when ear shoots were covered (Table 2). When ear shoots were uncovered, incidence decreased linearly with silk age (Fig. $2 \mathrm{~A}$ and Table 2). For covered ears of the hybrid Frontier, incidence decreased from about $70 \%$ when inoculated 2 days after silk emergence to $14 \%$ when ears were inoculated 15 days after silk emergence. For uncovered ears, incidence declined rapidly with silk age from $79 \%$ at silk emergence to $2 \%$ when ears were inocu-

Table 1. Regression equations describing the relationship between silk age and incidence of ears with galls of common smut on sweet corn hybrids when ear shoots were covered with shoot bags or uncovered prior to inoculation with Ustilago maydis in the 1995, 1996, and 1997 date-of-inoculation studies

\begin{tabular}{|c|c|c|c|c|c|c|c|c|c|}
\hline \multirow[b]{2}{*}{ Year $^{\text {v }}$} & \multirow[b]{2}{*}{ Hybrid } & \multirow[b]{2}{*}{ Silk treatment ${ }^{\mathrm{w}}$} & \multirow[b]{2}{*}{ Model } & \multicolumn{4}{|c|}{ Regression coefficients ${ }^{\mathrm{u}}$} & \multirow[b]{2}{*}{$r^{2 \times}$} & \multirow[b]{2}{*}{ CV $(\%)^{\mathrm{y}}$} \\
\hline & & & & $\mathbf{b}_{0}$ & $b_{1}$ & $\mathbf{b}_{2}$ & $\mathbf{b}_{3}$ & & \\
\hline \multirow[t]{2}{*}{1995} & Twelve hybrids & Ear shoots uncovered & Linear & $86.6 * * \mathrm{z}$ & $-6.8 * *$ & & & 0.86 & 29 \\
\hline & & Ear shoots covered & Quadratic & $90.4 * *$ & $-3.1 \mathrm{~ns}$ & $-0.35 * *$ & $\ldots$ & 0.86 & 28 \\
\hline \multirow[t]{2}{*}{ 1996/97 } & Dazzle & Ear shoots uncovered & Cubic & $50.3 * *$ & $23.3 * *$ & $-4.72 * *$ & $0.20 * *$ & 0.77 & 36 \\
\hline & & Ear shoots covered & Quadratic & $59.7 * *$ & $12.8 * *$ & $-1.39 * *$ & $\ldots$ & 0.77 & 25 \\
\hline \multirow[t]{2}{*}{$1996 / 97$} & Punchline & Ear shoots uncovered & Linear & $79.0 * *$ & $-8.8 * *$ & $\ldots$ & $\ldots$ & 0.78 & 45 \\
\hline & & Ear shoots covered & Linear & $89.7 * *$ & $-8.8 * *$ & $\ldots$ & $\ldots$ & 0.78 & 45 \\
\hline
\end{tabular}

u Coefficients of the regression equations describing the relationship between incidence of ears with galls and silk age (measured as days after silk emergence). Regression equations were calculated from 0 to 12 days after silk emergence in 1995, from 0 to 13 days after silk emergence for the hybrid Dazzle in 1996 and 1997, and from 0 to 11 days after silk emergence for Punchline in 1996 and 1997.

${ }^{v}$ Results for 1996 and 1997 were pooled because of no significant interaction of years with other terms in the analysis of variance combined over years.

w Primary ear shoots were covered with shoot bags prior to silk emergence until inoculation to prevent pollination or were uncovered to allow silks to pollinate normally. Shoot bags were not replaced after inoculation.

x $r^{2}=$ coefficient of determination.

${ }^{\mathrm{y}} \mathrm{CV}=$ coefficient of variation.

${ }^{\mathrm{z}} \mathrm{ns}, *$, and $* *$ indicate not significantly different from 0 at $P \geq 0.05$, significantly different from 0 at $P \geq 0.05$, and significantly different from 0 at $P \geq$ 0.01 , respectively, based on $t$ tests of regression coefficients. 
lated 16 days after silk emergence (Fig. 2B). Cubic regression models described 95 and $90 \%$ of the variation in incidence for uncovered and covered ears, respectively (Table 2). For the hybrid GH 2690, incidence was only $8 \%$ when ears were inoculated 3 days prior to silk emergence but about $55 \%$ when ears were inoculated 2 days after silk emergence (Fig. 2C). Incidence then decreased with silk age. Cubic regression models described 85 and $82 \%$ of the variation in incidence for uncovered ears and covered ears, respectively (Table 2). The hybrid Punchline was first inoculated 6 days after silk emergence in 1996
(Fig. 2D), when incidence was about $15 \%$ in all plots (Fig. 2D).

In 1997, incidence of ears with galls ranged from 0 to $100 \%$. Incidence varied with silk age, among hybrids, and between silk treatments. Mean incidence was $44 \%$ for the hybrid Dazzle, $31 \%$ for Frontier, $17 \%$ for GH 2690, and 26\% for Punchline. Mean incidence was $36 \%$ when ear shoots were covered and $23 \%$ when ear shoots were uncovered. Mean incidence was 62 , 32,16 , and $8 \%$ for the four silk ages determined by the fourth, third, second, and first planting dates, respectively. The interaction between silk age and silk treatments and the interaction between hybrids and silk treatments were significant. Incidence of ears with galls decreased with silk age for all hybrids in 1997 (Fig. 2E to H). Except for hybrid GH 2690, the decrease in incidence with silk age was more rapid when ear shoots were uncovered than when ear shoots were covered. For GH 2690, incidence did not differ between silk treatments (Fig. 2G).

For the hybrid Dazzle, incidence of ears with galls was 59\% when covered ears were inoculated 3 days prior to silk emergence and $83 \%$ when covered ears were inoculated 3 days after silk emergence

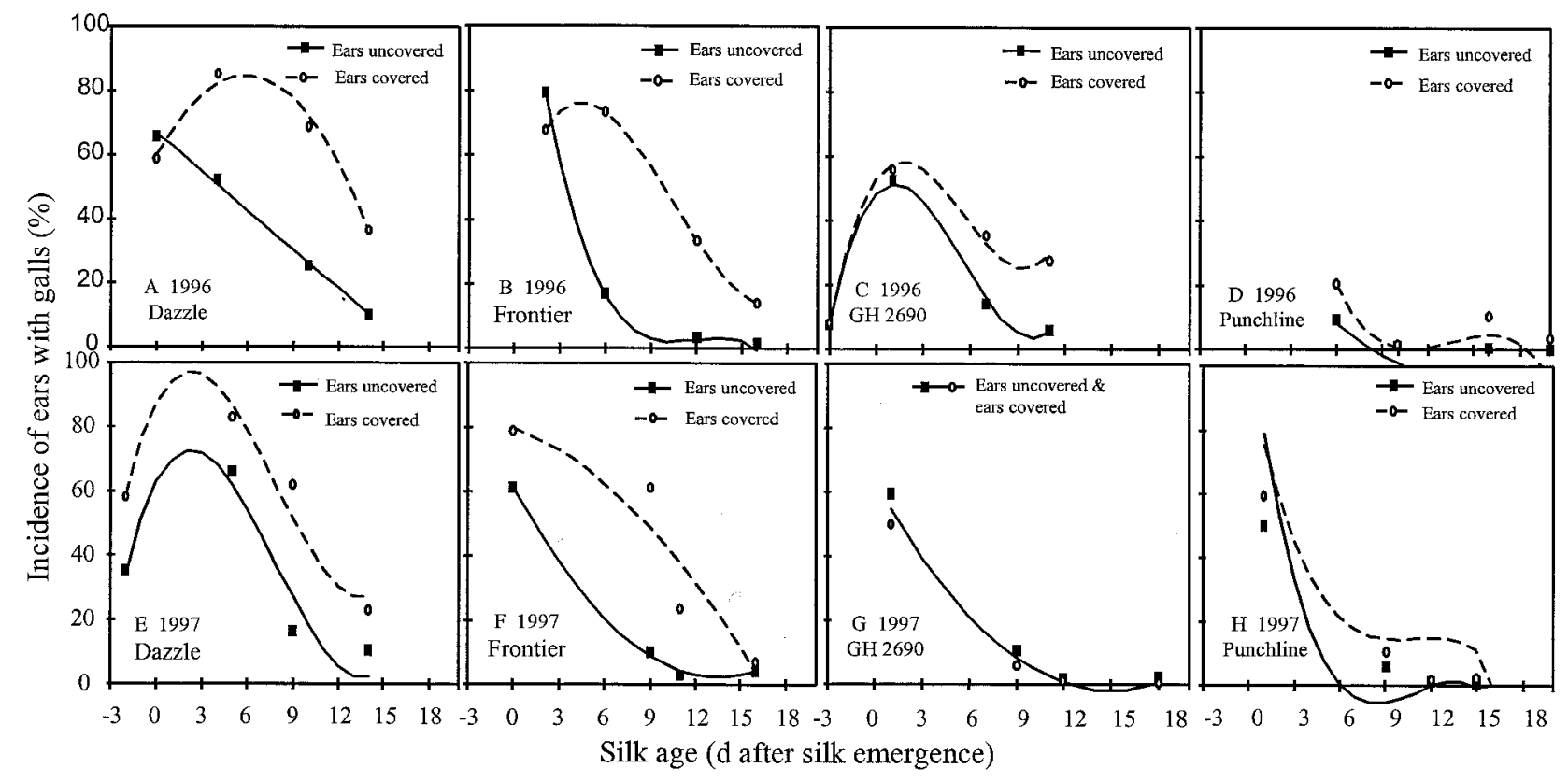

Fig. 2. Relationship between silk age and incidence of ears with galls of common smut on sweet corn when ear shoots were covered or uncovered prior to inoculation with Ustilago maydis in the date-of-planting studies for (A to D) 1996 and (E to H) 1997. Regression models are described in the text. Relationships are estimated using trend lines. Each data point is the mean of four replications.

Table 2. Regression equations describing the relationship between silk age and incidence of ears with galls of common smut on sweet corn hybrids when ear shoots were covered with shoot bags or uncovered prior to inoculation with Ustilago maydis in the 1996 and 1997 date-of-planting studies

\begin{tabular}{|c|c|c|c|c|c|c|c|c|c|}
\hline \multirow[b]{2}{*}{ Year } & \multirow[b]{2}{*}{ Hybrid } & \multirow[b]{2}{*}{ Silk treatment ${ }^{\mathrm{w}}$} & \multirow[b]{2}{*}{ Model } & \multicolumn{4}{|c|}{ Regression coefficients $^{v}$} & \multirow[b]{2}{*}{$r^{2 x}$} & \multirow[b]{2}{*}{$\mathrm{CV}(\%)^{\mathrm{y}}$} \\
\hline & & & & $\mathbf{b}_{\mathbf{0}}$ & $\mathbf{b}_{1}$ & $\mathbf{b}_{2}$ & $\mathbf{b}_{3}$ & & \\
\hline \multirow[t]{6}{*}{1996} & Dazzle & Ear shoots uncovered & Linear & $66.7 * * z$ & $-4.1 * *$ & & & 0.80 & 30 \\
\hline & & Ear shoots covered & Quadratic & $59.9 * *$ & $8.5 *$ & $-0.73 *$ & & 0.50 & 31 \\
\hline & Frontier & Ear shoots uncovered & Cubic & $139.0 * *$ & $-35.4 * *$ & $3.02 * *$ & $-0.08 *$ & 0.95 & 35 \\
\hline & & Ear shoots covered & Cubic & $45.3 * *$ & $15.4 *$ & $-2.20 *$ & $0.07 *$ & 0.90 & 20 \\
\hline & GH 2690 & Ear shoots uncovered & Cubic & $50.0 * *$ & $5.0 * *$ & $-2.56 * *$ & $0.16^{* *}$ & 0.85 & 46 \\
\hline & & Ear shoots covered & Cubic & $51.1 * *$ & $6.8 * *$ & $-2.13 * *$ & $0.12 * *$ & 0.82 & 29 \\
\hline \multirow{7}{*}{1997} & Dazzle & Ear shoots uncovered & Cubic & $62.8 * *$ & $9.1 * *$ & $-2.36 * *$ & $0.10 *$ & 0.67 & 41 \\
\hline & & Ear shoots covered & Cubic & $87.3 * *$ & $9.1 * *$ & $-2.36 * *$ & $0.10 *$ & 0.67 & 41 \\
\hline & Frontier & Ear shoots uncovered & Quadratic & $61.2 * *$ & $-8.7 * *$ & $0.32 * *$ & & 0.94 & 34 \\
\hline & & Ear shoots covered & Cubic & $78.9 * *$ & $35.3 * *$ & $-6.25 * *$ & $0.24 * *$ & 0.97 & 15 \\
\hline & GH 2690 & Ear shoots covered \& uncovered & Linear & $48.9 * *$ & $-3.2 * *$ & $\ldots$ & $\ldots$ & 0.68 & 86 \\
\hline & Punchline & Ear shoots uncovered & Linear & $62.7 * *$ & $-5.0 * *$ & $\ldots$ & $\ldots$ & 0.62 & 111 \\
\hline & & Ear shoots covered & Linear & $66.6 * *$ & $-4.2 * *$ & $\ldots$ & $\ldots$ & 0.71 & 50 \\
\hline
\end{tabular}

${ }^{\mathrm{v}}$ Coefficients of the regression equations describing the relationship between incidence of ears with galls and silk age (measured as days after silk emergence). Four silk ages were determined by four planting dates.

w Primary ear shoots were covered with shoot bags prior to silk emergence until inoculation to prevent pollination or were uncovered to allow silks to pollinate normally. Shoot bags were not replaced after inoculation.

${ }^{\mathrm{x}} r^{2}=$ coefficient of determination.

${ }^{y} \mathrm{CV}=$ coefficient of variation.

${ }^{\mathrm{z}} \mathrm{ns}, *$, and $* *$ indicate not significantly different from 0 at $P \geq 0.05$, significantly different from 0 at $P \geq 0.05$, and significantly different from 0 at $P \geq$ 0.01 , respectively, based on $t$ tests of regression coefficients. 
(Fig. 2E). Incidence for uncovered ears was $35 \%$ when ears were inoculated 3 days prior to silk emergence and $66 \%$ when ears were inoculated 3 days after silk emergence (Fig. 2E). Plots with covered ears had about $20 \%$ more ears with galls at all silk ages than plots with uncovered ears (Fig. 2E). A cubic regression model described $67 \%$ of the variation in incidence (Table 2). For the hybrid Frontier, incidence was $61 \%$ for uncovered ears and $79 \%$ for covered ears inoculated at silk emergence, and $4 \%$ for both treatments when inoculated 16 days after silk emergence (Fig. 2F). Quadratic and cubic regression models described 94 and $97 \%$ of the variation in incidence for uncovered and covered ears, respectively (Table 2). For the hybrid GH 2690, incidence decreased from about $55 \%$ when ears were inoculated at silk emergence to $<5 \%$ when inoculated 9 days after silk emergence (Fig. 2G and Table 2). A linear regression model described $68 \%$ of the variation in incidence with silk age. For the hybrid Punchline, incidence was about $55 \%$ for both silk treatments when inoculated 1 day after silk emergence. Incidence was $10 \%$ for covered ears and $6 \%$ for uncovered ears when inoculated 9 days after silk emergence (Fig. 2H).

\section{DISCUSSION}

Maize ears were susceptible to infection by $U$. maydis from silk emergence until 8 to 14 days after silk emergence. During this period, incidence of ears with galls decreased as silks aged, which has important implications in screening for resistance to common smut. Incidence of ears with galls differed by as much as $20 \%$ for plants inoculated 3 days apart. Incidence differed by as much as $70 \%$ when plants were inoculated a week apart. Thus, a hybrid that appears to be susceptible to common smut when inoculated within 2 or 3 days of silk emergence may appear to be relatively resistant if inoculated 10 days after silk emergence.

Inoculations with $U$. maydis must be timed precisely with silk emergence for an accurate assessment of reactions to common smut. Low levels of infection that make it difficult to differentiate hybrids can be avoided by inoculating ears between 2 and 4 days after silk emergence. Reid et al. (13) observed a similar period of susceptibility of maize ears to infection by $F$. graminearum. They stated that when silk inoculation is used to screen for resistance to $F$. graminearum, all plants should be inoculated at the same maturity, 4 to 7 days after silk emergence. Wells et al. (21) also observed a specific period of infection of 4 to 5 days for smut on pearl millet heads. They recommended inoculating between 0 and 3 days after head emergence to ensure adequate levels of smut.

The period of infection of maize ears by $U$. maydis was narrower and the suscepti- bility of ears to infection decreased more rapidly when silks were exposed to pollen than when silks were protected. Pollination reduced the period of infection by 1 to 4 days, and decreased incidence of ears with galls by as much as $50 \%$, compared to ears that were protected. Similar effects of pollination have been reported for other diseases. Higher levels of smut were observed on male-sterile lines of pearl millet compared to male-fertile lines, presumably due to the absence of pollen in male-sterile lines (21). Incidence of ergot on pearl millet pollinated $16 \mathrm{~h}$ after inoculation with Claviceps fusiformis Loveless was 7 to $21 \%$ compared to 60 to $80 \%$ for nonpollinated plants (17). Ergot resistance in certain lines of pearl millet appeared to be based on pollination-induced stigmatic constriction rather than host resistance (22). Lines thought to be resistant to ergot were as heavily infected as susceptible lines when grown under cool conditions that delayed anthesis. Similarly, common smut can be prevalent on hybrids thought to be resistant to $U$. maydis when anthesis and silk emergence do not coincide as a result of stressful growing conditions.

The relationship between incidence of ears with galls of common smut and silk age varied among hybrids, which may be associated with variation in silk emergence among hybrids. Silk emergence may occur over a longer period of time for some sweet corn hybrids than for others, with the main flush of silks emerging from husk leaves several days after initial silk emergence. A protracted period of silk emergence could extend the period of infection by $U$. maydis, because later-emerging silks are unpollinated and remain susceptible to infection. Although we did not monitor the nature of silk emergence among hybrids evaluated in our studies, this may explain the initial increase in incidence with silk age which we observed on the hybrids Dazzle and GH 2690.

Further studies are needed to determine how pollination reduces the period of infection of maize ears by U. maydis. Microscopic observations of hyphal growth on pollinated silks may determine whether abscission zone formation is the main factor limiting infection of the ovary when silks are pollinated. Maize mutants that do not shed silks after pollination also could be evaluated in microscopic studies of the interaction between maize pollen and $U$. maydis. Maize pollen stimulated germination of $F$. graminearum (Schwabe) in vitro on glass slides with pollen dialysate, on detached maize silks, and in vivo in leaf axils (9). Similar studies on the interaction between pollen on $U$. maydis would separate the effects of pollination on host tissue from the direct effects of pollination on the pathogen.

The results of this study demonstrate that precise timing of silk inoculation with silk maturity will result in more consistent responses of sweet corn hybrids to ear infection by $U$. maydis and would improve methods for screening for smut resistance. Poor timing of inoculation with silk maturity could result in susceptible lines being classified as resistant because they escape infection. In most commercial maize-breeding programs, hundreds to thousands of lines are screened for resistance to various diseases. Variation in maturity makes it difficult to inoculate each line at a precise stage of silk maturity. In addition, silk inoculation for common smut is labor intensive and occurs at pollination, when the demand for labor is greatest. This is compounded by sensitivity of silk-channel inoculations to variation in inoculation techniques among people, and to concentration of inoculum (2). These factors prohibit use of the silk-channel method of inoculating for common smut in largescale evaluations. The silk-channel inoculation method may be practical for evaluating a limited number of lines or for inducing ear galls for commercial production of huitlacoche (smut galls eaten at an immature stage; 18 ).

\section{ACKNOWLEDGMENTS}

We thank J. Manuel, B. Reep, and E. Swigart for assistance with field inoculations.

\section{LITERATURE CITED}

1. Christensen, J. J. 1963. Corn smut caused by Ustilago maydis. Am. Phytopathol. Soc. Monogr. 2.

2. du Toit, L. J. 1998. Field infection studies on common smut of sweet corn. Ph.D. thesis, University of Illinois, Urbana.

3. du Toit, L. J., and Pataky, J. K. 1996. Effects of inoculum concentration and pollination on incidence of ear galls of common smut on sweet corn. (Abstr.) Phytopathology 86:S63.

4. Enerson, P. M., and Hunter, R. B. 1980. A technique for screening maize (Zea mays L.) for resistance to ear mold incited by Gibberella zeae (Schw.) Petch. Can. J. Plant Sci. 60:1123-1128.

5. Headrick, J. M., Pataky, J. K., and Juvik, J. A. 1990. Relationships among carbohydrate content of kernels, condition of silks after pollination, and the response of sweet corn inbred lines to infection of kernels by Fusarium moniliforme. Phytopathology 80:487-494.

6. Heslop-Harrison, Y., Heslop-Harrison, J., and Reger, B. J. 1985. The pollen-stigma interaction in the grasses. 7. Pollen-tube guidance and the regulation of pollen tube number in Zea mays L. Acta Bot. Neerl. 34:193-211.

7. Heslop-Harrison, Y., Reger, B. J., and HeslopHarrison, J. 1984. The pollen-stigma interaction in the grasses. 5. Tissue organization and cytochemistry of the stigma ("silk") of Zea mays L. Acta Bot. Neerl. 33:81-99.

8. Marsh, S. F., and Payne, G. A. 1984. Preharvest infection of corn silks and kernels by Aspergillus flavus. Phytopathology 74:12841289.

9. Naik, D. M., and Busch, L. V. 1978. Stimulation of Fusarium graminearum by maize pollen. Can. J. Bot. 56:1113-1117.

10. Pataky, J. K., Nankam, C., and Kerns, M. R. 1995. Evaluation of a silk-inoculation technique to differentiate reactions of sweet corn hybrids to common smut. Phytopathology 85:1323-1328.

11. Platz, G. A. 1929. Some factors influencing 
the pathogenicity of Ustilago zeae (Beckm.) Unger. Iowa State Coll. J. Sci. 3:177-214.

12. Pope, D. D., and McCarter, S. M. 1992. Evaluation of inoculation methods for inducing common smut on corn ears. Phytopathology 82:950-955.

13. Reid, L. M., Bolton, A. T., Hamilton, R. I., Woldemariam, T., and Mather, D. E. 1992. Effect of silk age on resistance of maize to Fusarium graminearum. Can. J. Plant Pathol. 14:293-298.

14. Shurtleff, M. C., ed. 1980. Compendium of Corn Diseases, 2nd ed. Am. Phytopathol. Soc. St. Paul, MN.

15. Snetselaar, K. M., and Mims, C. W. 1993. Infection of maize stigmas by Ustilago may- dis: Light and electron microscopy. Phytopathology 83:843-850.

16. Steel, R. G. D., and Torrie, J. H. 1980. Principles and Procedures of Statistics, A Biometrical Approach, 2nd edition. McGraw-Hill, New York.

17. Thakur, R. P., and Williams, R. J. 1980. Pollination effects on pearl millet ergot. Phytopathology 70:80-84.

18. Valverde, M. E., Moghaddam, P. F., ZavalaGallardo, M. S., Pataky, J. K., Paredes-Lopez, O., and Pedersen, W. L. 1993. Yield and quality of huitlacoche on sweet corn inoculated with Ustilago maydis. HortScience 26:782785 .

19. Walter, J. M. 1934. The mode of entrance of
Ustilago zeae into corn. Phytopathology 24:1012-1020.

20. Walter, J. M. 1935. Factors affecting the development of corn smut, Ustilago zeae (Beckm.) Unger. Minn. Agric. Exp. Stn. Tech. Bull. 111:1-67.

21. Wells, H. D., Hanna, W. W., and Burton, G. W. 1987. Effects of inoculation and pollination on smut development in near-isogenic lines of pearl millet. Phytopathology 77:293296.

22. Willingale, J., Mantle, P. G., and Thakur, R. P. 1986. Postpollination stigmatic constriction, the basis of ergot resistance in selected lines of pearl millet. Phytopathology 76:536-539. 